\title{
Improved jet noise predictions in subsonic flows using an approximate composite asymptotic expansion of the adjoint Green's function in Goldstein's analogy
}

\author{
M. Z. Afsar* \\ Department of Mechanical \& Aerospace Engineering, University of Strathclyde, Glasgow, G1 I XJ, UK. \\ A. $\operatorname{Sescu}^{\dagger}$ \\ Department of Aerospace Engineering, Mississippi State University, Starkville, MS, USA. \\ S. J. Leib ${ }^{\ddagger}$ \\ Ohio Aerospace Institute, 22800 Cedar point road, Brookpark, OH 44142, USA.
}

\begin{abstract}
Our recent work on jet noise modeling (Afsar et al. 2019, PhilTrans. A., vol. 377) has confirmed that non-parallel flow effects are needed to determine the wave propagation aspect of the jet noise problem. The acoustic spectrum calculated using an asymptotic representation of non-parallel flow effects produces the correct spectral shape of the small angle radiation beyond that which can be predicted using a parallel (i.e. non-spreading) mean flow approximation to determine the wave propagation tensor in Goldstein's generalized acoustic analogy formulation. While the peak noise predicted using this approach works remarkably well at low frequencies (up to and slightly beyond the peak Strouhal number), the high frequency prediction in Afsar $e t$ al. (2019) relied upon an ad-hoc composite asymptotic formula for the propagator that was also restricted to the small angle spectra. In this paper we therefore attempt to remedy this defect by using the $O(1)$ frequency locally parallel flow Green's function as a kind-of outer solution to the propagator tensor in which the non-parallel flow theory used in the latter reference acts as the 'inner' solution that is valid at low frequencies and is transcendentally small beyond the peak frequency. The hope is that this approach will allow more robust high frequency predictions with a single set of turbulence parameters for the acoustic spectrum at any given acoustic Mach number. In other words, both non-parallel and locally parallel regions of the propagator tensor solution are multiplied by the same turbulence source structure in the acoustic spectrum integral.

The paper highlights the basic formalism of the low frequency jet noise theory and summarises the technical problems and strategy we use to extend this approach to higher frequencies.
\end{abstract}

\section{Introduction and qualitative considerations}

$\mathrm{R}$ ECENT advances in the modeling of low frequency noise radiated by an axi-symmetric air jet has highlighted the importance of including non-parallel mean flow effects in the lowest order asymptotic solution to the vector Green's function of the adjoint linearized Euler equations (ALEE). The solution to the latter set of equations enters the so-called propagator tensor in Goldstein's generalized formulation which itself is weighted by the Reynolds stress auto-covariance tensor in the acoustic spectrum integral formula for unheated flows. Afsar et al. 2019a, ([1], hereafter refered to as ASL19) found that a previously developed asymptotic theory of the ALEE, in which the jet spread rate $(\epsilon)$ is taken to be the same order as the non-dimensional frequency (i.e. the Strouhal number, $S t$ ) at the lowest order expansion of the solution, gave excellent predictive capability for the peak jet noise. More specifically, this distinguished limit which requires that the ALEE solution is determined when $S t \sim \epsilon$ had the correct low frequency roll-off as well as achieving the right magnitude for the peak sound observed at a polar observation angle, $\theta=30^{\circ}$ (in addition to angles above and below this location).

\footnotetext{
*Chancellor's Fellow. AIAA Member.

†Associate Professor, and AIAA Associate Fellow.

†Principal Scientist, AIAA Senior Member.
} 
Although this frequency where the non-parallel flow effects were found to enter the lowest order ALEE solution might be seen to be too low because spreadrates in jets are typically of the order of 0.1 (p.101ff. in Pope 2000), our calculations in ASL19 and Afsar et al. (2019b, [2]) showed that the limit of applicability typically extend to $S t \sim 0.5$ for high subsonic and supersonic acoustic Mach numbers (see Fig. 5b in ASL19 and Figs. 8b \& e in reference [2]). In the ASL19 predictions this limit of applicability was extended by constructing an approximate composite formula for the adjoint vector Green's function in which the non-parallel flow theory applies at low frequencies where the re-scaled frequency, $\Omega=\omega / \epsilon$, is held at $O(1)$, and the low frequency asymptotic solution to the parallel flow form of the ALEE (i.e. the Rayleigh equation) is used at Strouhal numbers beyond where the peak noise is observed. The ALEE solution then appears as the sum of the non-parallel flow and locally parallel flow models. Note that this avoids any possible double accounting of the overlap between both of these solutions because the non-parallel flow theory for the adjoint vector Green's function is exponentially small in the region of parameter space where $\Omega \gg O(1)$ and the low frequency asymptotic solution to the locally parallel flow equations is much smaller to the non-parallel flow Green's function at $\Omega=O(1)$ (by definition).

There were two pertinent technical problems with this type of 'composite expansion'. Firstly, that different turbulence scales were required in the solution region where $\Omega \gg O(1)$ and the sum of both non-parallel and parallel flow Green's functions where utilized. ASL19 did keep this to a suitable minimum (i.e. by only varying the transverse correlation length scale or the size of the anti-correlation region by tuning one parameter to be discussed in the appendix of this paper) in the auto-covariance tensor component $R_{1212}$. It should be appreciated that numerical experiments at $O(1)$ frequency and non-parallel flow show the acoustic spectrum for the peak jet noise involves only this component ([3] ). The second problem is that quite fundamentally, the use of the low frequency form of the parallel flow Green's function is not justified at these high frequencies where it would appear to be more consistent to use either a high frequency solution to the Rayleigh equation or the $O(1)$ frequency solution to the same equation.

The aim of this paper is, therefore, to investigate this problem with the aim of improving the prediction in this region of parameter space where $\Omega \rightarrow \infty$ under the particular limit such that $\omega=\epsilon \Omega=O(1)$. One of the effects of this will be to increase the prediction range of the unified jet noise model to observation angles greater than the peak noise angle of $\theta=30^{\circ}$. The rest of the paper is organized as follows. Section II summarizes the non-parallel flow theory for peak jet noise component of the acoustic spectrum analyzed in [1] \& [2]. We also discuss here the asymptotic structure of the latter solution approach in the parameter range of frequency $(S t)$, acoustic Mach number $(M a)$ and observation angle $(\theta)$. In §.III we summarize the parallel flow theory, valid at $\omega=O(1)$ and show how it can be used within the formalism of the non-parallel flow Green's function to formulate a more generalized composite asymptotic solution.

\section{Basic formalism of the generalized acoustic analogy}

Consider a region of non-homogeneous turbulence bounded within a high speed jet of order-1 acoustic Mach number, $M a=U_{J} / c_{\infty}$ and order-1 temperature ratio, $T R$. Pressure fluctuations within the jet propagate to the far field where they are perceived as sound. We use Goldstein's generalized acoustic analogy [4] to represent this process in a manner whereby the wave propagation is calculated via a propagator tensor that depends on ALEE solution. The acoustic spectrum also depends on the Reynolds stress auto-covariance tensor, which is modeled appropriately ([1] \& [2]). Let the pressure $p$, density $\rho$, enthalpy $h$, and speed of sound $c$ satisfy the ideal gas law equation of state $p=\rho c^{2} / \gamma$ and $h=c^{2} /(\gamma-1)$, where $\gamma$ denotes the ratio of specific heats.

The acoustic spectrum at the observation point, $\boldsymbol{x}=\left(x_{1}, \boldsymbol{x}_{T}\right)=\left(x_{1}, x_{2}, x_{3}\right)$, given by the Fourier transform

$$
I(\boldsymbol{x}, \omega) \equiv \frac{1}{2 \pi} \int_{-\infty}^{\infty} e^{i \omega \tau} \overline{p^{\prime}(\boldsymbol{x}, t) p^{\prime}(\boldsymbol{x}, t+\tau)} d \tau,
$$

of the far-field pressure auto-covariance, $\overline{p^{\prime}(\boldsymbol{x}, t) p^{\prime}(\boldsymbol{x}, t+\tau)}$, can be expressed as a volume integral over a unit volume of turbulence at $\boldsymbol{y}=\left(y_{1}, \boldsymbol{y}_{T}\right)=\left(y_{1}, y_{2}, y_{3}\right)$ in the jet via

$$
I(\boldsymbol{x} ; \omega)=\int_{V_{\infty}(\boldsymbol{y})} I(\boldsymbol{x}, \boldsymbol{y} ; \omega) d \boldsymbol{y},
$$

where, $V_{\infty}(y)$ is the entire source region. 
The pressure fluctuation in (1) is defined as $p^{\prime}(\boldsymbol{y}, \tau) \equiv p(\boldsymbol{y}, \tau)-\bar{p}(\boldsymbol{y})$ where over-bars are denote time average,

$$
\boldsymbol{\bullet}(\boldsymbol{x}) \equiv \lim _{T \rightarrow \infty} \frac{1}{2 T} \int_{-T}^{T} \bullet(\boldsymbol{x}, t) d t,
$$

such that $\bullet$ in (3) is a place holder for any fluid mechanical variable.

Goldstein \& Leib ([5]; hereafter referred to as G \& L) showed that $I(\boldsymbol{x}, \boldsymbol{y} ; \omega)$ on right side of (2) is given by formula,

$$
I(\boldsymbol{x}, \boldsymbol{y} ; \omega)=(2 \pi)^{2} \Gamma_{\lambda, j}(\boldsymbol{y} \mid \boldsymbol{x} ; \omega) \int_{V_{\infty}(\boldsymbol{\eta})} \Gamma_{\mu, l}^{*}(\boldsymbol{y}+\boldsymbol{\eta} \mid \boldsymbol{x} ; \omega) \mathcal{H}_{\lambda j \mu l}(\boldsymbol{y}, \boldsymbol{\eta} ; \omega) d \boldsymbol{\eta} .
$$

Here, asterisks denote complex conjugate and the Einstein summation convention is being used with the Greek tensor suffixes ranging from $(\lambda, \mu)=(1,2,3,4)$ and the Latin suffixes from $(i, j, k, l)=(1,2,3)$. The ALEE (defined below in 7) show that the Greens's function that enters (4) depends on the Favre-averaged mean flow field of the jet, $\tilde{\boldsymbol{v}}=\left\{\tilde{v}_{1}, \tilde{v}_{2}, \tilde{v}_{3}\right\}=\overline{\rho \boldsymbol{v}} / \bar{\rho}$ and mean speed of sound, $\widetilde{c^{2}}=\gamma \bar{p} / \bar{\rho}$, by the Fourier transform of the propagator tensor

$$
\Gamma_{\lambda, j}(\boldsymbol{y} \mid \boldsymbol{x} ; \omega) \equiv \Lambda_{\lambda \sigma, j}(\boldsymbol{y}) G_{\sigma}(\boldsymbol{y} \mid \boldsymbol{x} ; \omega):=\left(\delta_{\lambda \sigma} \frac{\partial}{\partial y_{j}}-(\gamma-1) \delta_{4 \sigma} \frac{\partial \tilde{v}_{\lambda}}{\partial y_{j}}\right) G_{\sigma}(\boldsymbol{y} \mid \boldsymbol{x} ; \omega)
$$

that involves an inner tensor product in suffix $\sigma$, of operator $\Lambda_{\lambda \sigma, j}(\boldsymbol{y})$, that spans $(4 \times 4 \times 3)$ dimensions corresponding to suffixes $(\lambda, \sigma, j)$ where comma after $j$ indicates that this suffix belongs to a derivative, and the first four components of the Fourier transform

$$
G_{\sigma}(\boldsymbol{y} \mid \boldsymbol{x} ; \omega)=\frac{1}{2 \pi} \int_{-\infty}^{\infty} e^{i \omega(t-\tau)} g_{\sigma 4}^{a}(\boldsymbol{y}, t-\tau \mid \boldsymbol{x}) d(t-\tau),
$$

of the five-dimensional adjoint vector Green's function, $g_{\sigma 4}^{a}(\boldsymbol{y}, \tau \mid \boldsymbol{x}, t)$, that appears on the left hand sides of the five ALEE (momentum, energy \& mass continuity) that were given previously in (4.8)-(4.10) of G\&L. The pressure-like Green's function component of $g_{\sigma 4}^{a}$ is subject to the strict causality condition $g_{44}^{a}(\boldsymbol{y}, t-\tau \mid \boldsymbol{x})=0$ for $t<\tau$ when $|\boldsymbol{x}| \rightarrow \infty$. The unit tensor in (5) is now a 4-dimensional Kronecker delta function and $G_{\sigma}(\boldsymbol{y} \mid \boldsymbol{x} ; \omega)$ is determined by the ALEE:

$$
\begin{aligned}
-D_{0} G_{i}+G_{j} \frac{\partial \tilde{v}_{j}}{\partial y_{i}}-\widetilde{c^{2}} \frac{\partial G_{4}}{\partial y_{i}}+(\gamma-1) \tilde{X}_{i} G_{4}-\frac{\partial G_{5}}{\partial y_{i}} & =0 \\
-D_{0} G_{4}-\frac{\partial G_{i}}{\partial y_{i}}+(\gamma-1) G_{4} \frac{\partial \tilde{v}_{i}}{\partial y_{i}} & =\frac{\delta(\boldsymbol{x}-\boldsymbol{y})}{2 \pi} \\
-D_{0} G_{5}+\tilde{X}_{i} G_{i} & =0,
\end{aligned}
$$

where $D_{0} \equiv i \omega+\tilde{\boldsymbol{v}}(\boldsymbol{y}) \cdot \boldsymbol{\nabla}$ is the convective derivative and $\boldsymbol{\nabla} \equiv\left\{\partial / \partial y_{1}, \partial / \partial y_{2}, \partial / \partial y_{3}\right\}$ is the three-dimensional gradient operator. The coefficients in 77 depend on the mean flow field through $\tilde{v}_{i}=\left(\tilde{v}_{1}, \tilde{v}_{2}, \tilde{v}_{3}\right) ; \widetilde{c^{2}}(\boldsymbol{y}) \equiv \gamma \bar{p} / \bar{\rho}$, the mean flow speed of sound squared, and

$$
\tilde{\boldsymbol{X}}(\boldsymbol{y})=(\tilde{\boldsymbol{v}} \cdot \boldsymbol{\nabla}) \tilde{\boldsymbol{v}},
$$

the mean flow advection vector.

The tensor, $\mathcal{H}_{\lambda j \mu l}(\boldsymbol{y}, \boldsymbol{\eta} ; \omega)$ in $(4)$ is related to the Fourier transform of the generalized auto-covariance tensor, $R_{\lambda j \mu l}(\boldsymbol{y}, \boldsymbol{\eta} ; \tau)$, of the stationary random function, $e_{\lambda j}(\boldsymbol{y}, \tau)=-\left[\rho v_{\lambda}^{\prime} v_{j}^{\prime}-\overline{\rho v_{\lambda}^{\prime} v_{j}^{\prime}}\right](\boldsymbol{y}, \tau)$, by the linear transformation $\mathcal{H}_{\lambda j \mu l}(\boldsymbol{y}, \boldsymbol{\eta} ; \omega):=\epsilon_{\lambda j \sigma m} H_{\sigma m \gamma n}(\boldsymbol{y}, \boldsymbol{\eta} ; \omega) \epsilon_{\mu l \gamma n}$ where $\epsilon_{\lambda j \sigma m} \equiv \delta_{\lambda \sigma} \delta_{j m}-\delta_{\lambda j} \delta_{\sigma m}(\gamma-1) / 2$ (see (5.12) to (5.13) in G \& L and Eqs. $2.9 \& 2.10$ in ASL19; note missing negative sign in the definition of $e_{\lambda j}$ below 2.10 in ASL19). The suffix ' 4 ' indicates enthalpy fluctuation via $v_{4}^{\prime}:=(\gamma-1)\left(h^{\prime}+v^{\prime 2} / 2\right) \equiv\left(c^{2}\right)^{\prime}+(\gamma-1) v^{\prime 2} / 2$ where $h^{\prime}$ is the fluctuating static enthalpy and $\left(c^{2}\right)^{\prime}$ is the fluctuations in the sound speed squared such that $v_{4}^{\prime} /(\gamma-1)$ denotes the moving frame stagnation enthalpy fluctuation [4].

The acoustic spectrum integral (2) is evaluated in cylindrical polar coordinates $\boldsymbol{y}=\left(y_{1}, r, \psi\right)$ with respect to an origin at the nozzle exit plane. Note this is commensurate with an axi-symmetric round jet for which we shall apply the new composite Green's function formula. Hence the mean flow field, commensurate with an axisymmetric jet, has components, $\boldsymbol{v}=\left(U, V_{r}\right)$. Moreover, if we let $\left(\boldsymbol{e}_{1}, \boldsymbol{e}_{r}, \boldsymbol{e}_{\phi}\right)$ be an orthogonal set of basis vectors in the cylindrical co-ordinate space, 
$\boldsymbol{G}=\left(G_{1}, G_{r}, G_{\phi}\right)$ in $7 \mathrm{7}$-c) $)$ can be expressed as a linear function of that basis by $\left(G_{i} \boldsymbol{e}_{i}\right) \boldsymbol{e}_{j}=G_{1} \delta_{j 1}+G_{r} \delta_{j r}+G_{\phi} \delta_{j \phi}$ where $\boldsymbol{G}=\left(G_{1}, G_{r}, G_{\phi}\right)$ are its respective components of $\boldsymbol{G}$ in the basis $\left(\boldsymbol{e}_{1}, \boldsymbol{e}_{r}, \boldsymbol{e}_{\phi}\right)$.

Before we perform any asymptotic analysis to determine the composite Green's function, we transform the independent and dependent variables in (7) by taking $U$ to be one of the independent variables of choice; i.e. under the one-to-one mapping $\left(y_{1}, r\right) \rightarrow\left(y_{1}, U\right)$ where $r \equiv\left|\boldsymbol{y}_{T}\right|=\sqrt{y_{2}^{2}+y_{3}^{2}}$ (see [6] and [2]). The latter reference showed that the ALEE in (7) can be transformed to the following mixed Partial Differential Equation (PDE) for the Green's function variable, $\tilde{v}=\tilde{v}\left(y_{1}, U\right) \equiv \widetilde{c}^{2} \tilde{G}_{4}+\tilde{G}_{5}$ :

$$
\mathcal{L} \tilde{v}\left(y_{1}, U\right)=\mathcal{F}(\tilde{\boldsymbol{S}}), \quad \downarrow
$$

for $\epsilon=O(1)$ spreadrates in which the Favre-averaged speed of sound $\widetilde{c^{2}}$ satisfies the Crocco-Busemann relation for a heated flow (Eq. 17 and discussion below Eq. 16 in [2]) or Crocco relation in an unheated flow [1]. The operator,

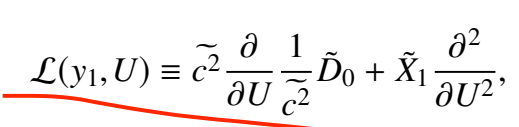

is hyperbolic and

$$
\mathcal{F}\left(\tilde{\boldsymbol{S}}_{)}\right) \mathcal{F}\left(\tilde{S}_{1}, \tilde{S}_{r}, \tilde{S}_{5}\right):=\tilde{S}_{1}-\left(\frac{\tilde{S}_{5}}{\widetilde{c^{2}}}+D_{0} \tilde{S}_{r}\right) .
$$

where $\left(\tilde{S}_{1}, \tilde{S}_{r}, \tilde{S}_{5}\right)$ are defined through Eqs. (21), (14) and the line below (15) in [2]. The components, $\tilde{S}=\left\{\tilde{S}_{1}, \tilde{S}_{r}, \tilde{S}_{5}\right\}$, are functions of the Green's function components $\left(\tilde{G}_{1}, \tilde{G}_{r}\right)$ in $(7)$ and mean flow field $\left(U, V_{r}\right)$ and the streanwise/radial components of the mean flow advection vector $\left(\tilde{X}_{1}, \tilde{X}_{r}\right)$, determined by $(8)$. Note that the tilde on the Green's function indicates its functional space is now $\left(y_{1}, U\right)$ via the implicit function theorem [6].

\section{Approximate uniformly valid solution for $\Gamma_{\lambda, j}$ in (5)}

When the temporal frequency is appropriately re-scaled as $\Omega=\omega / \epsilon=O(1)$ and considering the conditions across the surface $r=0$ in the $i=\phi$ component of $(77)$, reference [2] show that since the Green's function components, $\tilde{G}_{(r, \phi)}$, must remain bounded on the jet axis, $\tilde{G}_{(r, \phi)}=0$ at lowest order in $(7,, 9),(11$. Hence, the right hand side of 9 ) remains $O\left(\epsilon^{2}\right.$ (or at $o(1)$ relative to lowest order expansion) in the small jet spread rate limit $(\epsilon \ll O(1)$ - which an axisymmetric jet possess by definition). In other words, $\mathcal{F}(\boldsymbol{S})$ remains asymptotically sub-dominant in this limit. We summarise this next and show it leads to an asymptotic expansion of $\Gamma_{\lambda, j}$ that at its lowest order involves only a single term for the peak jet noise. In order to proceed with the asymptotic analysis as defined in ASL19 [1] we let all lengths be normalized by the $O(1)$ characteristic length scale, the nozzle diameter, $D_{J}$, and time scale $D_{J} / U_{J}$, where $U_{j}$ is the mean jet exit velocity. The fluid mechanical variables $(\tilde{\boldsymbol{v}}, p, \rho)$ may then be normalized by $U_{J}, \rho_{J} U_{J}^{2}$ and $\rho_{J}$.

Allowing the mean flow to vary over a slow streamwise length, $Y \equiv \epsilon y_{1}=O(1)$, corresponding to long streamwise length scales $y_{1}$, shows that it must expand according to (A.1-A.2) in G \& L [5]:

$$
\tilde{v}_{i}=\left\{U(Y), V_{r}(Y, U)\right\}= \begin{cases}U+\epsilon U^{(1)}(Y, U)+O\left(\epsilon^{2}\right), & i=1 \\ \epsilon\left(V_{r}+\epsilon V_{r}^{(2)}\right)(Y, U)+O\left(\epsilon^{3}\right), & i=r\end{cases}
$$

when $\widetilde{c^{2}}$ is determined by the Crocco-Busemann or Crocco relations. We have not put superscripts on the lowest order mean flow components, that would otherwise appear as $\left(U^{(0)}, V_{r}^{(1)}\right)$ respectively; they will be taken as that computed by the RANS solution. Moreover at this order in $\epsilon: \bar{\rho}(Y, U)=\rho(U)$ and $\bar{p}(Y, U)=$ const. and the mean flow advection vector, $X_{i}(\boldsymbol{y})$, that enters in $\tilde{S}_{i}=\left\{\tilde{S}_{1}, \tilde{S}_{r}, \tilde{S}_{5}\right\}$, similarly expands as

$$
\tilde{X}_{i}=\left\{\tilde{X}_{1}, \tilde{X}_{r}\right\}(Y, U)= \begin{cases}\epsilon \bar{X}_{1}(Y, U)+\epsilon^{2} \tilde{X}_{1}^{(2)}(Y, U)+O\left(\epsilon^{3}\right), & i=1 \\ \epsilon^{2} \bar{X}_{r}^{(2)}(Y, U)+O\left(\epsilon^{3}\right), & i=r\end{cases}
$$

where the leading streamwise term, $\bar{X}_{1}^{(1)} \equiv \bar{X}_{1}=V_{r}(\partial U / \partial r)$ and $\bar{X}_{r}^{(2)}=\left(U \partial / \partial Y+V_{r} \partial / \partial r\right) V_{r}$. Hence, measured from the jet centerline, the mean flow separates into an inner region, given by (12) \& (13), where (inner) radial co-ordinate $r=O(1)$, and an outer region where this expansion break downs; i.e., at large radial locations (with respect to inner variable, $r$ ) for which $R \equiv \epsilon r=O(1)$. Allowing $g_{v 4}^{a}(\boldsymbol{y}, \tau \mid \boldsymbol{x}, t)$ to depend on time, $\tau$, through the $O(1)$ slowly breathing time $\tilde{T}=\epsilon \tau$ allows mean flow non-parallelism to enter the lowest order asymptotic expansion of $\tilde{v}$ everywhere in the flow (and not just in the critical layer at supersonic speeds as in G\&L's solution) and at $M a=O(1)$. 
The scaled Fourier transform:

$$
\begin{aligned}
\tilde{v}(Y, U) & \equiv \frac{\epsilon}{4 \pi|\boldsymbol{x}|} e^{i \Omega X / c_{\infty}} \bar{v}\left(Y, U|X,| x_{T} \mid, 0 ; \Omega\right) \\
& =\frac{1}{2 \pi \epsilon} \int_{-\infty}^{\infty} e^{i \Omega\left(\tilde{T}_{0}-\tilde{T}\right)}\left(\tilde{c}^{2} \tilde{g}_{44}+\tilde{g}_{54}\right)\left(Y, U|X,| x_{T} \mid, 0 ; \tilde{T}_{0}-\tilde{T}\right) d\left(\tilde{T}_{0}-\tilde{T}\right),
\end{aligned}
$$

that is now determined by the homogeneous form of 9 at arbitrary $\Omega=\omega / \epsilon=O(1)$ frequencies when $\mathcal{F}(\tilde{\boldsymbol{S}})=o(1)$; i.e.,

$$
\mathcal{L} \bar{v}(Y, U) \equiv \widetilde{c^{2}} \frac{\partial}{\partial U}\left(\frac{1}{\widetilde{c^{2}}} \bar{D}_{0} \bar{v}\right)+\bar{X}_{1} \frac{\partial^{2} \bar{v}}{\partial U^{2}}=0, \text { for } \epsilon \ll O(1),
$$

where by the implicit function theorem, $\bar{v}\left(y_{1}, r\right) \equiv \bar{v}(Y, U) \equiv \widetilde{c}^{2} \bar{G}_{4}+\bar{G}_{5}$ is related to the zeroth-order azimuthal mode through the inverse Fourier transform of azimuthal expansion of $\tilde{v}$ in $(\Phi-\phi)$ (see Eq. $2.20 \& 2.21$ in [1]) where $\left(X, T_{0}\right)=\epsilon\left(x_{1}, t\right)$ are appropriate $O(1)$ slow variables at $\left(x_{1}, t\right)$.

Matching $v(Y, U)$ to the inner limit of the outer solution using Van Dyke's rule shows that it is uniquely determined by

$$
\begin{gathered}
\bar{v}(Y, 0) \rightarrow-i \Omega c_{\infty}^{2} e^{-i \Omega Y \cos \theta / c_{\infty}} \\
\frac{\partial \bar{v}}{\partial U}(Y, 0) \rightarrow-i \Omega c_{\infty} \cos \theta e^{-i \Omega Y \cos \theta / c_{\infty}}
\end{gathered}
$$

on the non-characteristic curve $U=0$, with $Y \geq 0$ (where, as indicated above, $U \rightarrow 0$ corresponds to outer limit, $r \rightarrow \infty)$. The coefficient $\bar{X}_{1}$ is the streamwise component of the mean flow advection vector (equation 5.15 in [6]) and $\bar{D}_{0}=i \Omega+U \partial / \partial Y$. Note the re-scaling of Fourier transform of $g_{v 4}^{a}(\boldsymbol{y}, \tau \mid \boldsymbol{x}, t)$ via a relation of the form of $\left.\sqrt{38}\right)$ above for the composite solution $\bar{v}=\widetilde{c^{2}} \bar{G}_{4}+\bar{G}_{5}$ determined by 15 or propagator 20 below that depends on $\left.\left(\bar{G}_{1}, \bar{G}_{4}\right), \bar{G}_{5}\right)$, is only necessary inasmuch as it simplifies matching conditions [16] \& (17).

\section{A. Propagator solution at $\omega / \epsilon=O(1)$ and $\epsilon \ll O(1)$}

Reference [1] show that the low-frequency acoustic spectrum at the observation point, $\boldsymbol{x}=\left(x_{1}, \boldsymbol{x}_{T}\right)=\left(x_{1}, x_{2}, x_{3}\right)$, due to momentum transfer by the fluctuating Reynolds stress and energy exchange (via temperature fluctuations in tensor $R_{\lambda j \mu l}(\boldsymbol{y}, \boldsymbol{\eta} ; \tau)$ when $\left.(\lambda, \mu)=4\right)$ in the acoustic spectrum formula 11 is given by

$$
I(\boldsymbol{x}, \boldsymbol{y} ; \omega) \approx\left(\frac{\epsilon}{2 c_{\infty}^{2}|\boldsymbol{x}|}\right)^{2}\left[4\left|G_{12}\right|^{2}+(3-\gamma) n_{2} \operatorname{Re}\left\{\Gamma_{41} G_{11}^{*}\right\}+n_{3}\left|\Gamma_{41}\right|^{2}\right] \Phi_{1212}^{*}
$$

where co-variance components $\left(R_{4111}, R_{4141}\right)$ are re-scaled such that $R_{4111}=n_{2} R_{1212}$ and $R_{4141}=n_{3} R_{1212}$ where $\left(n_{2}, n_{3}\right)$ are $O(1)$ constants. But [2] find that for the supersonic jets predictions using the momentum flux term in (18) alone (i.e. taking $n_{2}=n_{3}=0$ in 18 such that temperature associated correlation functions are negligible) gives accurate predictions up to $S t \sim 0.7-0.8$ for the heated jet spectra. This approximation was also confirmed in recent work by Gryazev et al. [7] for a co-axial jet at various operating points of nozzle exit Mach number and temperature ratio. Hence introducing this approximation shows that $I(\boldsymbol{x}, \boldsymbol{y} ; \omega)$ reduces to

$$
I(\boldsymbol{x}, \boldsymbol{y} ; \omega) \rightarrow\left(\frac{\epsilon}{c_{\infty}^{2}|\boldsymbol{x}|}\right)^{2}\left|G_{12}\right|^{2} \Phi_{1212}^{*}
$$

The Fourier transformed adjoint Green's functions $\left(\bar{G}_{1}, \bar{G}_{4}\right)$ represent the Green's function for the streamwise linearized momentum and energy equations of the generalized acoustic analogy ([4] \& [2]); they enter [18] through the propagator components:

$$
G_{12}=\tilde{G}_{12}(Y, U)=\frac{\partial \tilde{G}_{1}}{\partial r}-(\gamma-1) \tilde{G}_{4} \frac{\partial U}{\partial r}+O(\epsilon)
$$




\section{B. Propagator solution at $\omega=O(1)$ and $\epsilon \ll O(1)$}

1. Asymptotic structure of ALEE, (7), with $O(1)$ parameters of $(\omega, \theta, M a)$

Since the scaled frequency, $\Omega$, in $\sqrt{15}$ is $O(1)$ we may consider the what happens when $\Omega \rightarrow \infty$. In this case the overlap between the low frequency non-parallel flow solution to (7), given in the present context by Eqs. (15)-(17), and the parallel flow solution to (7), becomes apparent. More specifically, we keep $\epsilon=O(1)$ fixed in the definition of $\Omega \epsilon=\omega$, and let $\Omega \rightarrow \infty$ such that $\omega=O(1) \gg \epsilon$ ). In this case, inserting $\bar{D}_{0}=i \Omega+U \partial / \partial Y$ into 15 , and dividing through by $\Omega$ shows that

$$
\widetilde{c^{2}} \frac{\partial}{\partial U}\left[\frac{1}{\widetilde{c^{2}}}\left(1-i U \frac{\partial}{\partial \bar{Y}}\right) \bar{v}\right]+\frac{\bar{X}_{1}}{\Omega} \frac{\partial^{2} \bar{v}}{\partial U^{2}}=0
$$

non-parallelism is confined to a very thin streamwise region defined by the re-scaled co-ordinate $\bar{Y}=\Omega Y=O(1)$ of size $Y=O\left(\Omega^{-1}\right)$. In other words, the solution to $\bar{v}$ everywhere in $(Y, U)$ is determined by the locally parallel low frequency solution to (15). This is because the second term in (15), in which all non-parallel flow effects are effectively lumped together, is bounded by a $O(1 / \Omega)$ prefactor. Since this term remains algebraically small as $o(1)$ when $\Omega \rightarrow \infty, \bar{v}$ is then determined by a solution to the remaining term

$$
\widetilde{c^{2}} \frac{\partial}{\partial U}\left[\frac{1}{\widetilde{c^{2}}}\left(1-i U \frac{\partial}{\partial \bar{Y}}\right) \bar{v}\right]+O\left(\Omega^{-1}\right)=0
$$

Separating variables shows that $\bar{v}(Y, U)$

$$
\bar{v}(Y, U)=-\frac{i \Omega c_{\infty}^{2}}{1-\left(U / c_{\infty}\right) \cos \theta} e^{-i \bar{Y} \cos \theta / c_{\infty}}
$$

There is ample numerical confirmation of the sub-dominance of non-parallel flow effect in the large $\Omega$ (or, $\omega$ ) limit. See, for example, Fig. 16a in [3] where the acoustic analogy prediction based on a locally parallel flow Green's function solution is identical to the full numerical calculation of the adjoint equations for frequencies greater than $1000 \mathrm{~Hz}$ (i.e. for Strouhal numbers $>0.1$, the peak frequency); moreover, in Fig. 16b of [8], this overlap occurs at a later frequency of about $\gtrsim 0.3$ after re-scaling by $2 \pi$ to be consistent with our definition of Strouhal number.

In Fig. (1) below we calculate the non-parallel flow solution to (15) at $(\Omega, \theta)=\left(20,30^{\circ}\right)$ using the compressible form of the analytical mean flow field suggested by Tam and Burton [9]. This solution is then compared the parallel flow analytical formula (23). It is clear that the spatial structure of the $\bar{v}$-solution is almost identical with the magnitude differing by less $10 \%$.

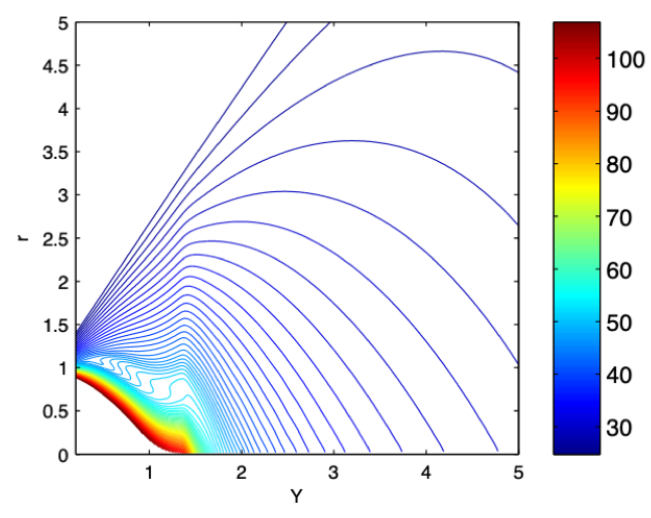

(a) Non-parallel flow solution to 15

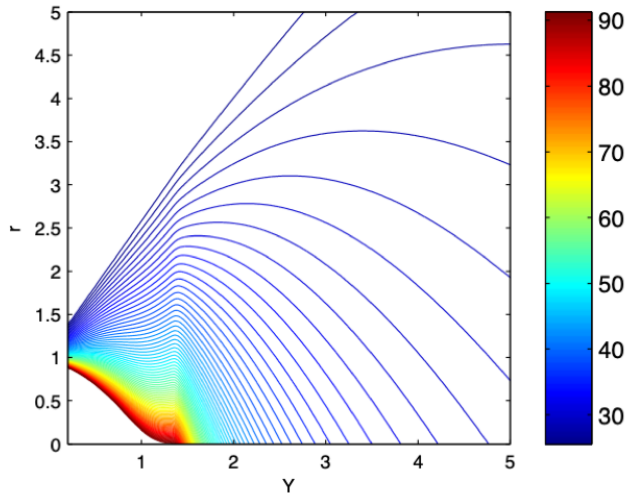

(b) Solution to 15 when $\bar{X}_{1}=0$ (Locally Parallel flow, Eq. 23

Fig. 1 Spatial structure of $|\bar{v}|$ in $(15)$ at $\Omega=20$ and $\theta=30^{\circ}$. 
2. Composite formula for $\bar{v}$ using $(23)$

ASL19 [1] used the fact that the acoustic spectrum depends on a parallel flow solution to the ALEE at high frequency to approximate the $\bar{v}$ solution in $\left(y_{1}, r ; \omega\right)$ co-ordinates as

$$
\bar{v}\left(y_{1}, r ; \omega\right) \rightarrow \bar{v}\left(y_{1}, r ; \omega\right)+H\left(\omega-\omega^{(0)}\right) \bar{v}^{p}\left(y_{1}, r ; \omega\right)
$$

where $\bar{v}^{p}\left(y_{1}, r ; \omega\right)$ is the locally parallel flow solution to $(15)$ given by $(23)$ and $\bar{v}\left(y_{1}, r ; \omega\right)$ is determined by solution to 15 and its matching conditions; $H(\bullet)$ is the Heaviside function of stated arguments and $\omega^{(0)}$, the peak frequency. Although (see Fig. 6 in ASL19) the acoustic predictions determined using the approximate composite Green's function formula (24) gave excellent agreement for the $30^{\circ}$ spectrum at all frequencies $0.01 \leq S t \leq 2.0$ for both SP07 $(M a, T R=0.9,0.84)$ and SP03 $(M a, T R=0.5,1.0)$, the turbulence parameters used in (4) at $S t \geq S t^{(0)}$ were not the same as frequencies below the peak. In order to avoid ad-hoc selection of the turbulence parameters, we now let $\bar{v}^{p}\left(y_{1}, r ; \omega\right)$ be the $O(1)$ parallel flow Green's function constructed next.

For a uni-directional transversely sheared mean flow where $\tilde{v}_{j}=\delta_{j 1} U\left(y_{2}, y_{3}\right), 77$ reduce to the following

$$
\begin{aligned}
\bar{D}_{0} G_{i}-G_{1} \frac{\partial U}{\partial y_{i}}+\widetilde{c^{2}} \frac{\partial G_{4}}{\partial y_{i}} & =0 \\
\bar{D}_{0} G_{4}+\frac{\partial G_{i}}{\partial y_{i}} & =-\frac{\delta(\boldsymbol{x}-\boldsymbol{y})}{2 \pi}
\end{aligned}
$$

where $\bar{D}_{0} \equiv i \omega+U\left(y_{2}, y_{3}\right) \partial / \partial y_{1}$. Since $\omega=O(1)$ the solution in the jet cannot be matched asymptotically to the outer zero-flow wave equation solution where the Delta function exists. It can be determined however by numerically patching both solutions together. The details appear in G \& L and Afsar [10]. The only difference with the presentation here is that we determine the correspondence between the appropriate Green's function variable used in these latter papers and $\bar{v}=\widetilde{c^{2}} G_{4}+G_{5}$.

Taking the streamwise convective derivative $\bar{D}_{0}$ of 25 ) and inserting the $i=1$ component 25 a) shows that

$$
\bar{D}_{0}^{2} G_{i}+\widetilde{c^{2}}\left[\frac{\partial U}{\partial y_{i}} \frac{\partial G_{4}}{\partial y_{1}}+\bar{D}_{0} \frac{\partial G_{4}}{\partial y_{i}}\right]=0
$$

But after inserting the commutative relation

$$
\bar{D}_{0} \partial G_{\lambda} / \partial y_{i}=\partial \bar{D}_{0} G_{\lambda} / \partial y_{i}-\left(\partial U / \partial y_{i}\right)\left(\partial G_{1} / \partial y_{1}\right)
$$

it follows that:

$$
\bar{D}_{0}^{2} G_{i}+\widetilde{c^{2}} \frac{\partial G_{0}}{\partial y_{i}}=0
$$

where $G_{0} \equiv \bar{D}_{0} G_{4}$ and we have used the fact that since 7 k) reduces to $\bar{D}_{0} G_{5}=0$, the space-time Green's function, $g_{5}(\boldsymbol{y}, \tau \mid \boldsymbol{x}, t)$ is purely convected, i.e., $g_{5}=g_{54}^{a}\left(\tau-y_{1} / U ; \boldsymbol{y}\right)$. After taking Fourier transforms in the $(t-\tau)$ and the streamwise direction $\left(x_{1}-y_{1}\right)$ shows that $(U k-\omega) \hat{G}_{5}=0$ and therefore that the space-time Fourier transform, $\hat{G}_{5}$, must be zero at all $(\omega, k)$ other than at the critical layer where $(U k-\omega)=0$.

The solution to the vector Green's function, $G_{\lambda}(\boldsymbol{y} \mid \boldsymbol{x} ; \omega)$ where $\lambda=(1,2,3,4)$ reduces to solving Eqs. 28) and $25 \mathrm{p})$ for $G_{i}(\boldsymbol{y} \mid \boldsymbol{x} ; \omega)$ and $G_{0}(\boldsymbol{y} \mid \boldsymbol{x} ; \omega)$. But since a uni-directional transversely sheared mean flow $\tilde{v}_{j}=\delta_{j 1} U\left(y_{2}, y_{3}\right)$ is idnependent of the streamwise direction, the Green's function $G_{\lambda}(\boldsymbol{y} \mid \boldsymbol{x} ; \omega)$ depends on streamwsie direction only in difference $\left(x_{1}-y_{1}\right)$ owing to the right hand side of $\left.25 \mathrm{~b}\right)$. The field equations for $G_{i}$ and $G_{0}$ therefore reduce to solving:

$$
\begin{aligned}
(U k-\omega)^{2} \hat{G}_{i}-\widetilde{c^{2}} \frac{\partial \hat{G}_{0}}{\partial y_{i}} & =0 \\
\hat{G}_{0}+\frac{\partial \hat{G}_{i}}{\partial y_{i}} & =-\frac{\delta\left(\boldsymbol{x}_{T}-\boldsymbol{y}_{T}\right)}{(2 \pi)^{2}}
\end{aligned}
$$

at a radial location (measured from the centerline) in the jet in which $r=\sqrt{y_{2}^{2}+y_{3}^{2}}=O(1)$ and where

$$
\hat{G}_{\lambda}\left(\boldsymbol{y}_{T} \mid \boldsymbol{x}_{T} ; \omega, k\right)=\frac{1}{2 \pi} \int_{-\infty}^{\infty} e^{-i k\left(x_{1}-y_{1}\right)} G_{\lambda}\left(x_{1}-y_{1}, \boldsymbol{y}_{T}, \omega \mid \boldsymbol{x}_{T}\right) d\left(x_{1}-y_{1}\right)
$$


$\hat{G}_{i}$ in Eq. 29p) can obviously be eliminated using 29 ) to give the Rayleigh equation for $\hat{G}_{0}$ :

$$
\hat{G}_{0}+\frac{\partial}{\partial y_{i}}\left\{\frac{\widetilde{c^{2}}}{(U k-\omega)^{2}} \frac{\partial \hat{G}_{0}}{\partial y_{i}}\right\}=-\frac{\delta\left(\boldsymbol{x}_{T}-\boldsymbol{y}_{T}\right)}{(2 \pi)^{2}} .
$$

We have taken liberties of using the gradient operator symbol, to refer to the Fourier transform of the same operator, namely $\partial / \partial y_{i}=-i k \delta_{i 1}+\delta_{i 2} \partial / \partial y_{2}+\delta_{i 3} \partial / \partial y_{3}$ in Cartesian co-ordinates. Inserting the latter then shows that (31) takes the the form of the generalized Sturm-Louvillie

$$
\frac{\partial}{\partial y_{i}}\left\{\frac{\widetilde{c^{2}}}{(U k-\omega)^{2}} \frac{\partial \hat{G}_{0}}{\partial y_{i}}\right\}+\left[1-\frac{k^{2} \widetilde{c^{2}}}{(U k-\omega)^{2}}\right] \hat{G}_{0}=-\frac{\delta\left(\boldsymbol{x}_{T}-\boldsymbol{y}_{T}\right)}{(2 \pi)^{2}}, \quad j=(2,3) .
$$

Our Green's function, $\hat{G}_{0}=-i(U k-\omega) \hat{G}_{4}$, governed by 32 , is identical to Eq. (4.21) in G \& L such that $g_{44}^{a}=-\bar{D}_{0}^{2} g_{a} / D \tau^{2}$ where $g_{a}$ is the adjoint Lilley equation Green's function (note that the negative sign is missing in Eq. 4.16 in $\mathrm{G} \& \mathrm{~L}[5])$. For a parallel flow

$$
v(\boldsymbol{y}, \tau \mid \boldsymbol{x}, t)=\widetilde{c^{2}} g_{44}^{a}(\boldsymbol{y}, \tau \mid \boldsymbol{x}, t)+g_{54}^{a}(\boldsymbol{y}, \tau \mid \boldsymbol{x}, t) \rightarrow \widetilde{c^{2}} g_{44}^{a}
$$

since $g_{54}^{a}=0$ in this case. The solution for $G_{4}$ in the zero-flow wave equation region far outside of the jet shows that the latter Fourier transformed solution has a prefactor that goes like $i \omega /(4 \pi|x|) c_{\infty}^{2}$. Since $G_{0} \equiv \bar{D}_{0} G_{4}$, taking the inverse Fourier transform of the solution to (32) as

$$
G_{\lambda}\left(\boldsymbol{y}_{T} \mid \boldsymbol{x}_{T} ; \omega, x_{1}-y_{1}\right)=\int_{-\infty}^{\infty} e^{i k\left(x_{1}-y_{1}\right)} \hat{G}_{\lambda}\left(\boldsymbol{y}_{T} \mid \boldsymbol{x}_{T} ; \omega, k\right) d k
$$

using (5.21), (5.22) \& (5.23) in [5] shows that

$$
G_{0}\left(\boldsymbol{y}_{T} \mid \boldsymbol{x}_{T} ; \omega, x_{1}-y_{1}\right) \rightarrow \sum_{n=-\infty}^{\infty} e^{i n(\phi-\Phi)} \int_{-\infty}^{\infty} e^{i k\left(x_{1}-y_{1}\right)} \hat{G}_{0}^{(n)}\left(\boldsymbol{y}_{T} \mid \boldsymbol{x}_{T} ; \omega, k\right) d k .
$$

where

$$
\hat{G}_{0}^{(n)}\left(\boldsymbol{y}_{T} \mid \boldsymbol{x}_{T} ; \omega, k\right)=\frac{e^{-x_{T} \sqrt{k^{2}-k_{\infty}^{2}}}}{\sqrt{x_{T}}} \frac{w_{n}^{(2)}(r, k)}{\Delta_{n}}
$$

and

$$
\mathcal{L}_{n} \hat{G}_{0}^{(n)}\left(\boldsymbol{y}_{T} \mid \boldsymbol{x}_{T} ; \omega, k\right)=\frac{\delta(r-R)}{(2 \pi)^{3} r}
$$

in a cylindrical polar co-ordinate space where the Fourier transform of the gradient operator is now $\partial f(r, \phi ; k) / \partial y_{i}=$ $\left(i k \delta_{i 1}+\delta_{i r} \partial / \partial r+\delta_{i \phi} \partial / \partial r \phi\right) f(r, \phi ; k)$ where $(r, \phi)$ are the radial and azimuthal directions respectively.

Then (7.2) \& (7.11) in G \& L and the method of stationary phase shows that $\left(\sin \theta \Delta_{n}\right)=-k_{\infty}^{2} /\left[(2 \pi)^{3}|\boldsymbol{x}| W_{n}\left(w_{n}^{(1)}, w_{n}^{(2)}\right)\right]$ when $\Delta_{n}$ (and therefore $W_{n}$ via Eq. 7.9 in G \& L [5]) is evaluated at infinity.

$$
\begin{aligned}
& G_{0}\left(\boldsymbol{y}_{T} \mid \boldsymbol{x}_{T} ; \omega, x_{1}-y_{1}\right) \equiv \\
& G_{0}\left(r \mid R ; \omega, x_{1}-y_{1}, \phi-\Phi\right) \rightarrow-e^{-i k_{\infty}\left(y_{1} \cos \theta-|x|\right)} \frac{k_{\infty}^{2}}{(2 \pi)^{3}|\boldsymbol{x}|} \bar{G}_{0}(r \mid R ; \omega, \phi-\Phi)
\end{aligned}
$$

where $w_{n}^{(2)}$ that enters the scaled solution, $\bar{G}_{0}(r \mid R ; \omega, \phi-\Phi)$ given by:

$$
\bar{G}_{0}(r \mid R ; \omega, \phi-\Phi)=\sum_{n=-\infty}^{\infty} e^{i n(\phi-\Phi)} \frac{w_{n}^{(2)}}{W_{n}\left(w_{n}^{(1)}, w_{n}^{(2)}\right)}
$$


is determined by solution to $\mathcal{L}_{n} w_{n}^{(2)}=0$ defined by:

$$
\begin{aligned}
\mathcal{L}_{n} w_{n}^{(2)}= & \left(\frac{1}{r} \frac{d}{d r}\left\{\frac{r\left(\widetilde{c}^{2} / c_{\infty}^{2}\right)}{(1-M(r) \cos \theta)^{2}} \frac{d}{d r}\right\}\right. \\
& \left.+k_{\infty}^{2}\left[1-\frac{\left(\widetilde{c}^{2} / c_{\infty}^{2}\right)}{(1-M(r) \cos \theta)^{2}}\left(\cos ^{2} \theta+\frac{n^{2}}{k_{\infty}^{2} r^{2}}\right)\right]\right) w_{n}^{(2)}=0 .
\end{aligned}
$$

To avoid differentiating mean flow in the Rayleigh equation [40, it is easier to solve 29]. Taking the inverse transform of 297 in the jet region therefore shows that:

$$
\begin{aligned}
& \frac{d w_{n}^{(2)}}{d r}=\frac{k_{\infty}^{2}}{a_{r}^{2}}(1-M(r) \cos \theta)^{2} \tilde{g}_{r}^{(n)} \\
& \frac{d \tilde{g}_{r}^{(n)}}{d r}=\frac{a_{r}^{2}}{(1-M(r) \cos \theta)^{2}}\left[\cos ^{2} \theta+\frac{n^{2}}{k_{\infty}^{2} r^{2}}-1\right] w_{n}^{(2)}-\frac{\tilde{g}_{r}^{(n)}}{r}
\end{aligned}
$$

where $a_{r}^{2}=\tilde{c}^{2} / c_{\infty}^{2}$ and $\tilde{g}_{r}^{(n)}$ is the inverse Fourier transform of the $\hat{G}_{r}$ that is bounded at $r=0$. Note that Eqs. 41p can be easily manipulated to recover (40). That is, re-arranging 41 a) for $d r \tilde{g}_{r}^{(n)} /(r d r)$ inserting this into 41 a) and re-arranging after multiplying through by $k_{\infty}^{2}$ gives 40 .

In the neighborhood around the regular singular point, $r=0$, the mean flow is nearly constant and the homogeneous form of Rayleigh equation 32 in $r$ reduces to a Bessel equation for $\hat{G}_{4}(\boldsymbol{y} \mid \boldsymbol{x} ; \omega)$; hence $\hat{G}_{4}^{n} \sim J_{n} \rightarrow r^{n}$ by the method of Frobenious (Abramowitz \& Stegun 1963, p.360; see also Tam \& Auriault 1998). Hence $w_{n}^{(2)}(r) \& \tilde{g}_{r}^{(n)}$ in 441 \& b), possess the following initial conditions:

$$
\begin{aligned}
& w_{n}^{(2)}\left(r_{\text {start }}\right)=-i \omega\left(1-M\left(r_{\text {start }}\right) \cos \theta\right) r_{\text {start }}^{n} \text { and } \\
& \tilde{g}_{r}^{(n)}\left(r_{\text {start }}\right)=-\frac{i \omega}{\left(k_{\infty}^{2}\right) / a_{r}^{2}\left(r_{\text {start }}\right)\left(1-M\left(r_{\text {start }}\right) \cos \theta\right)} n r_{\text {start }}^{n-1}
\end{aligned}
$$

where $r_{\text {start }}$ is the starting grid point closest to $r=0$ in the radial direction.

The other homogeneous solution $w_{n}^{(1)}$ that enters the Wronskian formula is when $\hat{G}_{4}^{n} \sim H_{n}^{(1)}$ at $r \rightarrow \infty$, which is bounded at $r_{\text {end }}$ in the numerical solution. Since $M\left(r_{\text {end }}\right)=0$, the homogeneous solution $w_{n}^{(1)}$ must go like $-i \omega H_{n}^{(1)}$

has the far-field behavior

$$
w_{n}^{(1)} \rightarrow \frac{-i \omega}{\sqrt{r}} e^{-k_{\infty} r \sqrt{\cos ^{2} \theta-1}}=\frac{-i \omega}{\sqrt{r}} e^{-i k_{\infty} r \sin \theta} \text { as } r \rightarrow \infty .
$$

The latter and the numerical solution to (40) is used to compute the Wronskian

$$
W_{n}\left(w_{n}^{(1)}, w_{n}^{(2)}\right)=w_{n}^{(1)} \frac{d}{d r} w_{n}^{(2)}-w_{n}^{(2)} \frac{d}{d r} w_{n}^{(1)}
$$

where $w_{n}^{(1)}(r)$ is given by 43 and its derivative is

$$
\frac{d w_{n}^{(1)}(r)}{d r} \rightarrow \frac{i \omega}{\sqrt{r}}\left[i k_{\infty} \sin \theta+\frac{1}{r}\right] e^{-i k_{\infty} r \sin \theta} \text { as } r \rightarrow \infty .
$$

and $w_{n}^{(2)}(r)$ and its derivative $d w_{n}^{(2)}(r) / d r$ is found using the numerical solution to 40 as $r \rightarrow \infty$.

Since $v=\widetilde{c^{2}} g_{44}^{a}$ and $\bar{D}_{0} \tilde{v}=\widetilde{c^{2}} \bar{D}_{0} G_{4}=\widetilde{c^{2}} G_{0}, G_{0}=\left(1 / c^{2}\right) \bar{D}_{0} \tilde{v}$ where

$$
G_{0}(r \mid R ; \omega)=\frac{1}{2 \pi} \int_{-\infty}^{\infty} e^{i \omega(t-\tau)} g_{0}^{a}(r \mid x ; t-\tau) d(t-\tau),
$$

Taking the space-time Fourier transform of $\sqrt{33}$ and using the method of stationary phase shows that this latter correspondence between Fourier transformed variables, $\hat{v}$ and $\hat{G}_{0}$ is

$$
\hat{G}_{0}\left(\boldsymbol{y}_{T} \mid \boldsymbol{x}_{T} ; \omega, k\right)=-i \frac{(U k-\omega)}{\widetilde{c^{2}}} \hat{v}\left(\boldsymbol{y}_{T} \mid \boldsymbol{x}_{T} ; \omega, k\right)
$$


and therefore that

$$
G_{0}\left(r \mid R ; \omega, x_{1}-y_{1}, \phi-\Phi\right)=i \frac{k_{\infty}}{c_{\infty}} \frac{(1-M(r) \cos \theta)}{\left(\widetilde{c^{2}} / c_{\infty}^{2}\right)} \tilde{v}\left(r \mid R ; \omega, x_{1}-y_{1}, \phi-\Phi\right)
$$

Inserting 38$)$ into this shows that

$$
\begin{gathered}
\tilde{v}\left(\boldsymbol{y}_{T} \mid \boldsymbol{x}_{T} ; \omega, x_{1}-y_{1}\right) \equiv \\
\tilde{v}^{p}\left(r \mid R ; \omega, x_{1}-y_{1}, \phi-\Phi\right) \rightarrow \frac{i k_{\infty}}{(2 \pi)^{3}|\boldsymbol{x}|} e^{-i k_{\infty}\left(y_{1} \cos \theta-|\boldsymbol{x}|\right)} \bar{v}(r \mid R ; \omega, \phi-\Phi)
\end{gathered}
$$

where the scaled solution $\bar{v}(r \mid R ; \omega, \phi-\Phi)$ is now given by:

$$
\bar{v}(r \mid R ; \omega, \phi-\Phi)=\frac{c_{\infty}\left(\widetilde{c^{2}} / c_{\infty}^{2}\right)}{(1-M(r) \cos \theta)} \bar{G}_{0}(r \mid R ; \omega, \phi-\Phi)
$$

where $\bar{G}_{0}(r \mid R ; \omega, \phi-\Phi)$ is given by 39$)$.

We can write (49) with the same prefactor as (2.21) in [1]. Note the error in their equation since the prefactor for $\bar{v}$ should be proportional to $1 /(4 \pi|x|)$ after using $[33$ and the Fourier transform of Eq. (4.7) in [6]. Hence we may write 49 as follows

$$
\begin{gathered}
\tilde{v}\left(\boldsymbol{y}_{T} \mid \boldsymbol{x}_{T} ; \omega, x_{1}-y_{1}\right) \equiv \\
\tilde{v}\left(r \mid R ; \omega, x_{1}-y_{1}, \phi-\Phi\right) \rightarrow \frac{\epsilon}{4 \pi|\boldsymbol{x}|} e^{i k_{\infty}|x|} \bar{v}^{p}(r \mid R ; \omega, \phi-\Phi)
\end{gathered}
$$

where $\bar{v}^{p}(r \mid R ; \omega, \phi-\Phi)$ is given by

$$
\bar{v}^{p}(r \mid R ; \omega, \phi-\Phi)=\frac{i \Omega}{2 \pi^{2}} e^{-i k_{\infty} y_{1} \cos \theta} \frac{\left(\widetilde{c^{2}} / c_{\infty}^{2}\right)}{(1-M(r) \cos \theta)} \bar{G}_{0}(r \mid R ; \omega, \phi-\Phi)
$$

where as in the previous relation, $\bar{G}_{0}(r \mid R ; \omega, \phi-\Phi)$ is given by 39 .

The analysis in $\S . I V$ can be summarized in the diagrams of the asymptotic structure of the Fourier transformed vector Green's function, $G_{\sigma}(\boldsymbol{y} \mid \boldsymbol{x} ; \omega)$ that satisfies ALEE, 7 . Here we illustrate how the structure of $G_{\sigma}(\boldsymbol{y} \mid \boldsymbol{x} ; \omega)$ maps when the $O(1)$ parameters of $(\omega, \theta, M a)$ are appropriately varied over their respective ranges.

\section{Conclusion}

In this paper we have developed a jet noise model that should allow the peak jet noise predictions shown in ASL19 [1] to be extended to $O(1)$ frequencies. As opposed to the latter, we aim to accomplish this by constructing a composite expansion for the Fourier transformed adjoint vector Green's function solution to (7) that uses the $O(1)$ frequency parallel flow (i.e. the Rayleigh equation) solution at higher frequencies. The hope is that this will enable the same turbulence parameters to be used at all frequencies; i.e. at low frequencies where the Green's function is determined by solution to the non-parallel flow asymptotic equation 15 and at $O(1)$ frequencies where the Rayleigh equation solution (40) comes into play.

\section{Acknowledgments}

Computational resources from HPC2, Mississippi State University, are appreciated. MZA would like to thank Strathclyde University for financial support from the Chancellor's Fellowship.

\section{References}

[1] Afsar, M. Z., Sescu, A., and Leib, S. J., "Modelling and prediction of the peak-radiated sound in subsonic axisymmetric air jets using acoustic analogy-based asymptotic analysis," Phil. Trans. R. Soc. A, Vol. 377, 2019.

[2] Afsar, M. Z., Sescu, A., and Sassanis, V. G., "Effect of non-parallel mean flow on the acoustic spectrum of heated supersonic jets: Explanation of "jet quietening"," Phys. Fluids, Vol. 31, 105107, 2019. 
[3] Karabasov, S. A., Afsar, M. Z., Hynes, T. P., Dowling, A. P., McMullan, W. A., Pokora, C. D., Page, G. J., and McGuirk, J. J., "Jet noise: acoustic analogy informed by Large-Eddy Simulation," AIAA J., Vol. 48, 2010, p. 1312.

[4] Goldstein, M. E., “A generalized acoustic analogy,” J. Fluid Mech., Vol. 488, 2003, p. 315.

[5] Goldstein, M. E., and Leib, S. J., “The Aero-acoustics of slowly diverging supersonic jets,” J. Fluid Mech., Vol. 488, 2008, p. 315.

[6] Goldstein, M. E., Sescu, A., and Afsar, M. Z., "Effect of non-parallel mean flow on the Green's function for predicting the low-frequency sound from turbulent air jets," J. Fluid Mech., Vol. 695, 2012, p. 199.

[7] Gryazev, V., Markesteijn, A. P., and Karabasov, S. A., "Low-Order models of dual-stream jet noise with temperature effects based on the Goldstein generalised acoustic analogy," 25th AIAA Aeroacoustics Conference, 2019.

[8] Karabasov, S. A., Bogey, C., and Hynes., "Computation of noise of initially laminar jets using a statistical approach for the acoustic analogy: application and discussion,” AIAA 2011-2929, 2011.

[9] Tam, C. K. W., and Burton, D., "Sound generated by instability waves in supersonic flows, Part 2," J. Fluid Mech., Vol. 138, 1984, p. 273

[10] Afsar, M. Z., "Asymptotic properties of the overall sound pressure level of sub-sonic air jets using isotropy as a paradigm," $J$. Fluid Mech., Vol. 664, 2010, p. 510. 


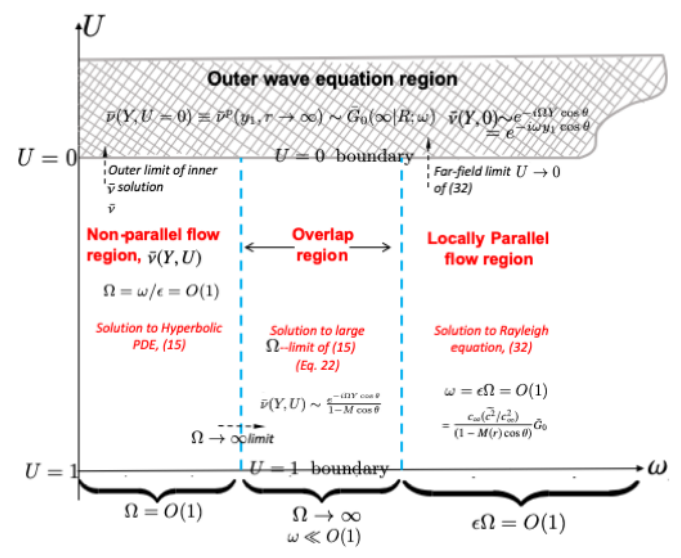

(a) Variation of 77 with frequency, $\omega$ at fixed $(\theta, M a) \approx\left(30^{\circ}, 0.9\right)$

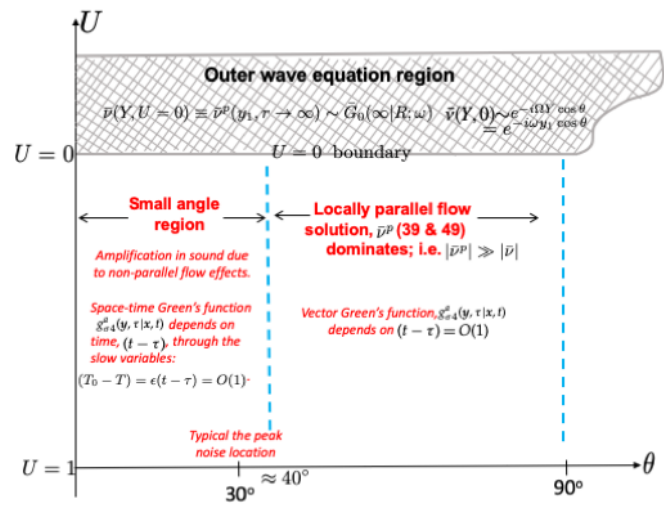

(b) Variation of 77 with frequency, $\theta$ at fixed $(S t, M a) \approx(0.2,0.9)$

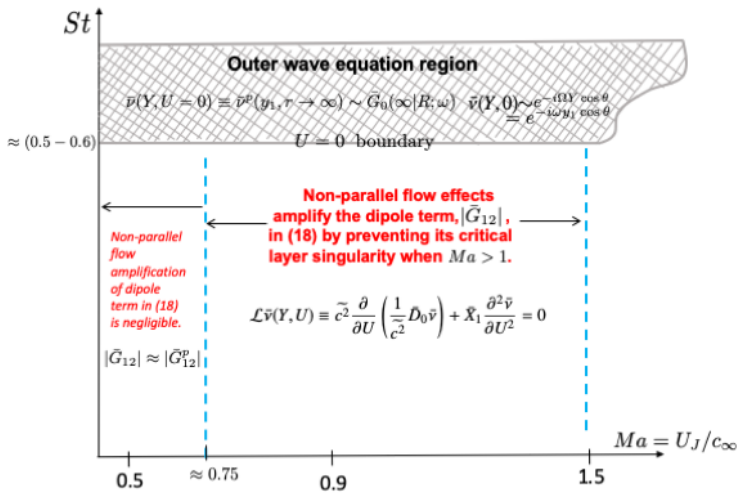

(c) Variation of (7) with acoustic Mach number, $M a$ at fixed $(S t, \theta) \approx\left(0.2,30^{\circ}\right)$

Fig. 2 Asymptotic structure of the ALEE (7) with $O(1)$ parameters $(\omega, \theta, M a)$ for small jet spreadrates, $\epsilon \ll O(1)$. 